\title{
Lack of Preemptive Analgesia by Intravenous Flurbiprofen in Thyroid Gland Surgery: A Randomized, Double-blind and Placebo-controlled Clinical Trial
}

\author{
Zhaodi Zhang ${ }^{1,}$, , Haifang Zhao ${ }^{1, *}$, Changsong Wang2 ${ }^{2}$, Fei Han ${ }^{1}$, Guonian Wang ${ }^{1 凶}$ \\ 1. Department of Anesthesiology, the Third Affiliated Hospital of Harbin Medical University, Harbin, Heilongjiang 150081, China. \\ 2. Department of Anesthesiology, the First Affiliated Hospital of Harbin Medical University, Harbin, Heilongjiang 150001, China \\ * Zhaodi Zhang and Haifang Zhao contributed equally to this work.
}

$\triangle$ Corresponding author: Prof. Guonian Wang, Department of Anesthesiology, the Third Affiliated Hospital of Harbin Medical University, Harbin, Heilongjiang 150081, China. (Fax: 86-0451-86298811; Email: wangguonian609cn@yahoo.com.cn)

(C) Ivyspring International Publisher. This is an open-access article distributed under the terms of the Creative Commons License (http://creativecommons.org/ licenses/by-nc-nd/3.0/). Reproduction is permitted for personal, noncommercial use, provided that the article is in whole, unmodified, and properly cited.

Received: 2011.03.28; Accepted: 2011.07.07; Published: 2011.07.15

\begin{abstract}
Background Nowadays, increasingly more preemptive analgesia studies focus on postoperative pain; however, the impact of preemptive analgesia on perioperative opioid requirement is not well defined. This study was carried out in order to evaluate whether preoperative intravenous flurbiprofen axetil can reduce perioperative opioid consumption and provide postoperative analgesia in patients undergoing thyroid gland surgery.

Methods Ninety patients undergoing elective thyroid gland surgery were randomly assigned to three groups. Group A (Control) was administered Intralipid ${ }^{\circledR} 2 \mathrm{ml}$ as a placebo 15 min before the cervical plexus block and at the end of the surgery; Group B (Routine analgesia) was administered a placebo $15 \mathrm{~min}$ before the cervical plexus block and flurbiprofen $50 \mathrm{mg}$ at the end of the surgery; Group C (Preemptive analgesia) was administered intravenous flurbiprofen $50 \mathrm{mg} 15 \mathrm{~min}$ before the cervical plexus block and a placebo at the end of the surgery. Sufentanil administration during the surgery and the $24 \mathrm{~h}$ satisfaction score on analgesic therapy were both recorded. The analgesic efficacy was assessed at 1, 2, 4, 6, 8, 12, and 24 hours after the surgery, based on visual analog scales.

Results Ninety patients were involved in the study. One patient from Group B did not have their scheduled surgery; eighty-nine patients completed the study. There were no significant differences in the patient demographics between the three groups. Visual analog scales: 1, 2, $4 \mathrm{~h}$ for Group A was significantly higher than Groups B and C $(P<0.05)$; Sufentanil administration during surgery: Group $C$ was obviously lower compared to Groups A and B $(P<0.05) ; 24 \mathrm{~h}$ satisfaction score: Groups B and C were higher than Group A $(P<0.05)$.

Conclusion Preoperative administration of intravenous Flurbiprofen axetil reduced analgesic consumption during surgery, but not postoperative pain scores.
\end{abstract}

Key words: preemptive analgesia; Flurbiprofen; thyroid gland surgery; cervial plexum block; postoperative pain.

\section{Introduction}

Preemptive analgesia is the administration of an analgesic before a painful stimulus that prevents the establishment of the altered processing of afferent input, which amplifies postoperative pain; and effec- 
tive preemptive analgesia should prevent the establishment of central sensitization caused by incisional and inflammatory injuries (covers the period of surgery and the initial postoperative period). ${ }^{1}$ Experimental evidences suggest that better postoperative analgesia in patients receiving the analgesic preoperatively compared to those patients, who were treated postoperatively..$^{2-4}$ Except postoperative pain, perioperative analgesic consumption may be another index of preemptive analgesia, because it can indicate peripheral and central sensitization during surgery indirectly and it has a direct effect on postoperative pain. Unfortunately, perioperative analgesic consumption was ignored in preemptive analgesia research. Maybe, that is one of the reasons that some preemptive analgesia research reached negative findings. 5,6

Flurbiprofen axetil (FA) is an injectable nonselective COX inhibitor, with a high affinity to inflammatory tissues because of composed emulsified lipid microspheres. 7,8 Preoperative intravenous administration of flurbiprofen reduces postoperative pain after tonsillectomy, spinal fusion surgery, hysterectomy, and arthroscopic rotator cuff repair surgery.9-12 However, there are a few reports on whether preoperative FA can reduce perioperative opioid consumption and postoperative pain after thyroid gland surgery. In this study, the hypothesis that preoperative administration FA reduces perioperative opioid consumption and provides postoperative analgesia for patients undergoing thyroid gland surgery, as compared with postoperative administration FA or placebo was tested.

\section{Methods}

This prospective, double-blind, randomized study was approved by the Ethics Committee of the Harbin Medical University and informed consent was obtained from the patients prior to study enrollment. Ninety patients undergoing elective thyroid gland surgery were involved in this study.

The inclusion criteria were: 1) ASA physical status I or II patients undergoing elective thyroid surgery; 2) aged 30-60 years. The exclusion criteria were: 1) patients who had received nonsteroidal anti-inflammatory drugs (NSAIDs), opioid or drugs with known analgesic properties in the $24 \mathrm{~h}$ before surgery; 2) patients with a history of allergic reaction to local anesthetics, opioid, NSAIDs; 3) patients with any contraindications for the use of NSAIDs, such as: gastrointestinal ulcer, coagulation disorders, renal dysfunction, heart failure and ischemic heart disease; 4) patients unable to comprehend the concept of the visual analog pain scale (VAS). All of the patients were instructed the day before surgery about the study protocol and particularly about the use of VAS. No premedication was given, and all the patients fasted from midnight before surgery.

On arrival at the operating room, patients received 2-3 mg intravenous midazolam. The standard monitors, including pulse oximetry, electrocardiography, and noninvasive arterial blood pressure, were applied. Patients were randomized to treatment groups A (Control), B (Routine analgesia), or C (Preemptive analgesia) in a sequence generated by a computerized random number generator and sealed in numbered, opaque envelopes. The envelopes contained two 5-mL syringes, labeled "pre" and "post," with the contents blinded to anesthesiology, surgeons, operating room staff, recovery room staff, and the patient until the study was completed. Group A (Control) received Intralipid ${ }^{\circledR} 2 \mathrm{ml}$ as a placebo $15 \mathrm{~min}$ before the cervical plexus block and at the end of the surgery; Group B (Routine analgesia) received a placebo 15 min before cervical plexus block and flurbiprofen axetil $50 \mathrm{mg}(2 \mathrm{ml})$ at the end of surgery; Group C (Preemptive analgesia) received intravenous flurbiprofen axetil $50 \mathrm{mg} 15 \mathrm{~min}$ before the cervical plexus block and a placebo at the end of the surgery.

Bilateral combined superficial and deep cervical plexus block with $0.5 \%$ ropivacaine was given in all the cases. A deep cervical plexus block was performed by using a 23-gauge, short beveled needle (Pole, Top, Japan). It was inserted behind the lateral border of the sternocleidomastoid muscle, $3 \mathrm{~cm}$ distal to the mastoid process. After negative aspiration for blood, $8 \mathrm{~mL}$ of solution was injected. The same needle was also used in a superficial cervical plexus block, and it was inserted at the midpoint of the sternocleidomastoid muscle, corresponding to the C3 transverse apophysis. After negative aspiration for blood in three directions, $4.5 \mathrm{~mL}$ of the solution was injected up and down at the posterior border of the sternocleidomastoid muscle to block the occipital, auricular, and supraclavicular branches of the superficial cervical plexus and $1.5 \mathrm{~mL}$ was injected horizontally above the muscle to block the transverse cervical nerve. Mean arterial blood pressure was maintained within $20 \%$ of the baseline values, in which additional boluses sufentanil were given in incremental doses of 1-2 $\mathrm{gg}$ when necessary. All surgical and anesthetic procedures were performed by the same teams.

Postoperative pain management was standardized as follows: For postoperative pain relief, tramadol was administered in increments of $50 \mathrm{mg}$ on patient demand with a lock-out time of $4 \mathrm{~h}$ and a maximum dose of $300 \mathrm{mg} /$ day. One ward nurse, who was blinded to group allocation, documented the 
postoperative pain intensity using VAS at the first rescue analgesics request, and at 1, 2, 4, 6, 8, 12, and 24 hours after surgery at rest. The time to the first request and the number of times tramadol was used in the first 24 hours after surgery were recorded. If the patients experienced severe nausea and vomiting, $10 \mathrm{mg}$ metoclopramide iv was administered. Sufentanil consumption during surgery and $24 \mathrm{~h} 4$-point rating scale satisfaction score $(0$ very unsatisfied, 1 unsatisfied, 2 satisfied, 3 very satisfied) on analgesic therapy were both recorded blindly by one of the authors. Side effects related to the regional anesthetic technique, such as cervical epidural analgesia and diaphragmatic palsy, were recorded. Particularly, patients were clinically evaluated by an experienced anesthesiologist for respiratory distress related to bilateral diaphragmatic palsy at recovery and in the PACU. Should this occur, dynamic chest radiograph examination would be performed to ascertain the diagnosis. Other side effects associated with flurbiprofen, such as vomiting and antiemetic requirements, were recorded.

Statistical analyses were performed using a statistical software package (SPSS13.0, Chicago, IL). Data were analyzed by the analysis of variance (ANOVA) with repeated measures, using one dependent variable on the time course. Analysis of the categorical data and proportions was performed using the $\chi^{2}$ test. The differences between the two groups were evaluated using the Student $\mathrm{t}$ test, Mann-Whitney rank sum test, and Fisher exact test, where appropriate. $P<0.05$ was considered significant. The sample size was calculated to detect a difference of 2.0 in pain intensity on a visual analogue scale (VAS 0-10). Based on the assumption of a standard deviation of 2.0, we calculated a sample size of 20 patients per group. This number would be sufficient to find the mentioned clinical endpoint with a power of 0.89 as statistically significant.

\section{Results}

Ninety patients were involved in the study. One patient from the group B did not have her scheduled surgery; eighty-nine patients completed the study. The demographic characteristics and intraoperative data of the three groups are presented in Table 1 . There were no significant differences in the patient demographics between the three groups. In addition, pulse oximetry, heart rates, blood pressures, blood loss, and fluid administration during surgery were not statistically different between the groups $(P>0.05)$.
Table 1. Characteristics of the Patients

\begin{tabular}{llll}
\hline Variable & $\begin{array}{l}\text { Control } \\
(\mathrm{n}=30)\end{array}$ & $\begin{array}{l}\text { Routine anal- } \\
\text { gesia } \\
(\mathrm{n}=29)\end{array}$ & $\begin{array}{l}\text { Preemptive anal- } \\
\text { gesia } \\
(\mathrm{n}=30)\end{array}$ \\
\hline Gender (M/F) & $18 / 12$ & $16 / 13$ & $17 / 13$ \\
Age (yrs) & $47.43 \pm 6.35$ & $49.80 \pm 7.24$ & $47.93 \pm 6.94$ \\
Weight $(\mathrm{kg})$ & $59.73 \pm 6.32$ & $62.45 \pm 7.28$ & $59.84 \pm 7.50$ \\
$\begin{array}{l}\text { Height }(\mathrm{cm}) \\
\begin{array}{l}\text { Duration of sur- } \\
\text { gery (min) }\end{array}\end{array}$ & $174 \pm 8$ & $172 \pm 6$ & $171 \pm 6$ \\
\hline
\end{tabular}

There were no significant differences between the demographic variables for the groups. Data are mean $\pm \mathrm{SD}$ or $\mathrm{n}$.

VAS data are presented in Fig. 1. VAS in group B and $\mathrm{C}$ were significantly lower than that in group $\mathrm{A}$ at $1,2,4 \mathrm{~h}$ after surgery $(P<0.05)$. There were no differences in VAS between Groups A, B and C at 6, 8, 12, 24 $\mathrm{h}$ after surgery $(P>0.05)$. The number of patients who need additional postoperative analgesia in Group $A$ is more than Groups B and C $(P<0.05)$ (Fig. 2).

Sufentanil consumption during surgery are presented in Fig. 3, in which sufentanil consumption in group C $(3.68 \pm 1.20 \mu \mathrm{g})$ was obviously lower compared to Groups A $(6.40 \pm 1.66 \mu \mathrm{g})$ and B $(7.21 \pm$

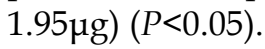

Patients evaluated the overall quality of their postoperative analgesia in the recovery process using the $24 \mathrm{~h}$ satisfaction score, both Group B $(1.82 \pm 1.03)$ and Group C $(1.75 \pm 0.89)$ were higher than Group A $(0.85 \pm 0.93)(P<0.05)$ (Fig. 4$)$.

No patient showed any adverse effect associated with FA and there were no surgical complications.

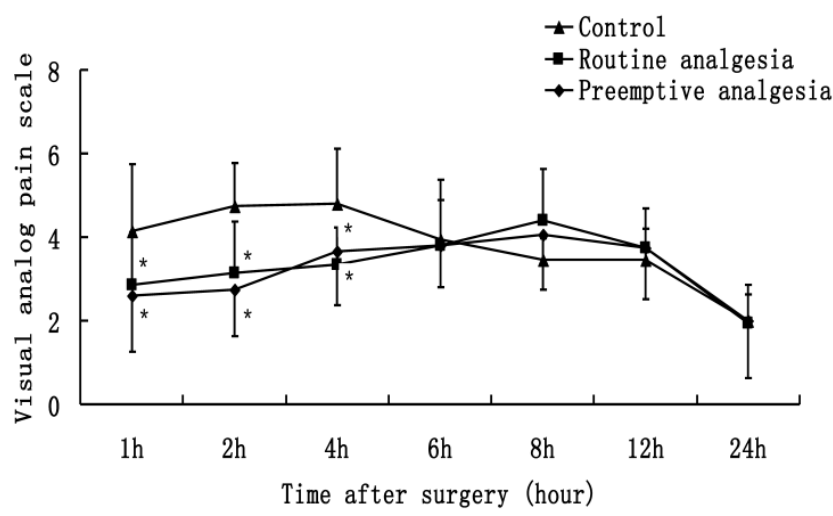

Figure 1. Visual analog pain scale, patients rated their levels of pain on the $0-10 \mathrm{cmVAS}(0 \mathrm{~cm}=$ no pain to $10 \mathrm{~cm}$ $=$ the worst possible pain). ${ }^{*} P<0.05$ Preemptive analgesia and routine analgesia group versus the control group. 


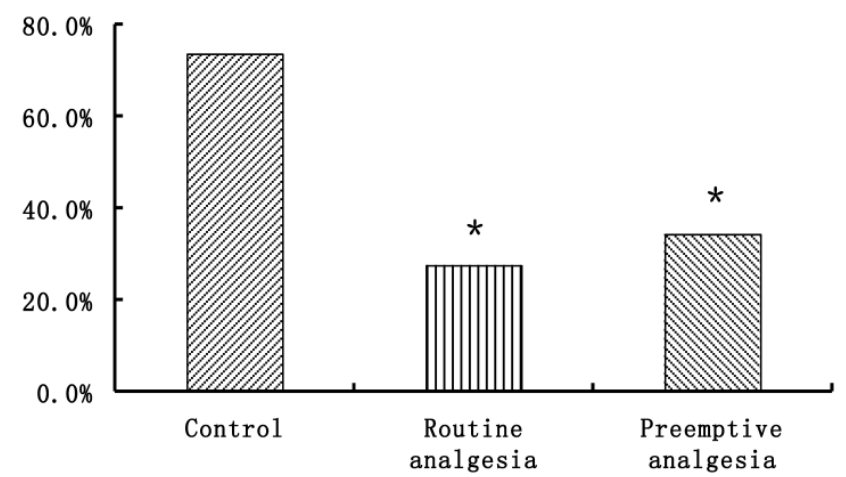

Figure 2. The ratios of people need additional postoperative analgesia. ${ }^{*} P<0.05$ Preemptive analgesia and routine analgesia group versus the control group.

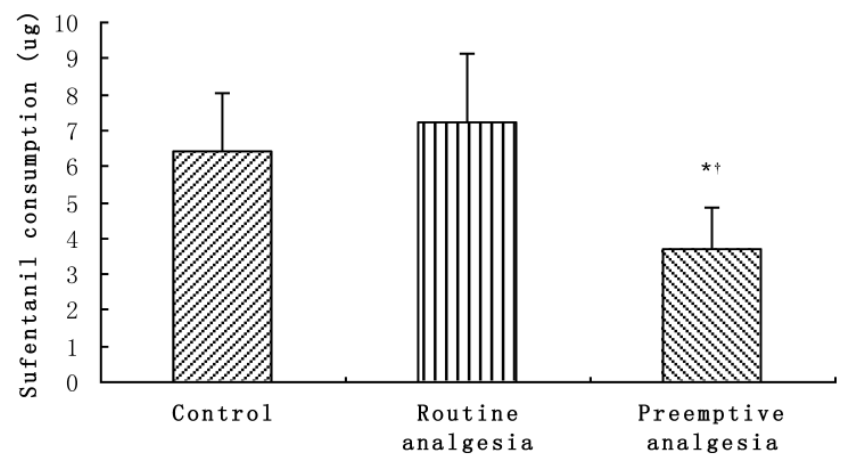

Figure 3. Sufentanil consumption during surgery. $P<0.05$, Preemptive analgesia group versus the control group; ${ }^{\dagger} P<0.05$ Preemptive analgesia group versus routine analgesia group.

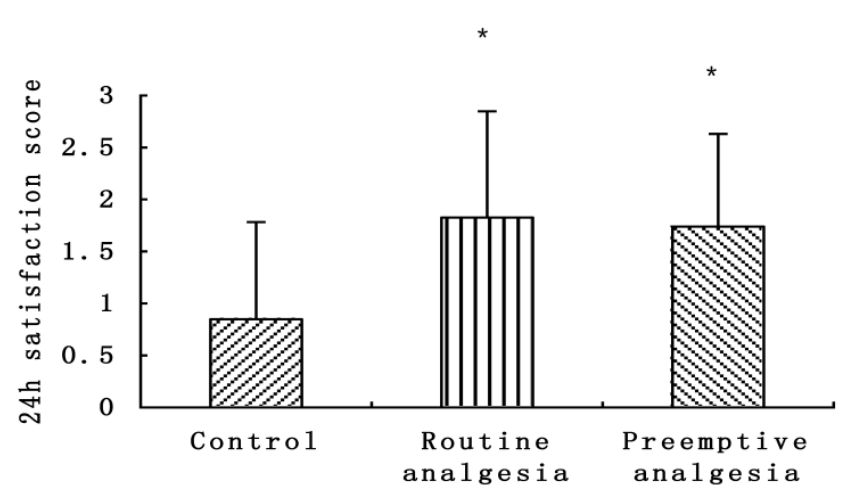

Figure 4. $24 \mathrm{~h}$ satisfaction score, patients rated their levels of analgesic satisfaction on the 0 -4score ( 0 very unsatisfied, 1 unsatisfied, 2 satisfied, 3 very satisfied). * $P<0.05$ Preemptive analgesia and routine analgesia group versus the control group.

\section{Discussion}

The present results indicate that preoperative FA provides less sufentanil consumption during surgery, better immediate postoperative analgesia than placebo. But, compared to patients receiving FA at the end of surgery, there is lack of preemptive analgesia effect.

In animal experiments, the validity of preemptive analgesia has been demonstrated. ${ }^{33,14}$ Nevertheless, some clinical studies have conflicting results regarding the efficacy of preemptive analgesia. ${ }^{15-17} \mathrm{~A}$ meta-analysis published in 2002 showed that there is no conclusive clinical evidence to support preemptive analgesia. ${ }^{18}$ However, another meta-analysis published in 2005 has shown that preemptive local anesthetic wound infiltration and nonsteroidal anti-inflammatory drug (NSAID) administration improved analgesic consumption and the time to the first rescue analgesic request, but not postoperative pain scores. ${ }^{19}$

Some authors have suggested that the effects of preemptive analgesia may vary according to the type of surgery. ${ }^{20}$ Whether preemptive analgesia can be effective depends on the prevention of the establishment of central sensitization. In some kinds of surgery, such as fracture, spinal disc herniation, appendicitis, and acute or chronic pain already exist. Under these clinical conditions, we can easily notice that central sensitization has already been established by presurgical pain. ${ }^{21}$ To avoid this condition, we chose thyroid gland surgery, which is not to be studied in preemptive analgesia research and there is no presurgical pain.

In general, general anesthesia is more suitable for thyroid gland surgery. In this study, we carried out a bilateral combined superficial and deep cervical plexus block. The most important reason is that we wanted to compare the preemptive analgesia effect on sufentanil consumption during surgery. Perioperative analgesic consumption may be another index except postoperative pain, because it can indicate periphery and central sensitization during surgery and it has a direct effect on postoperative pain. ${ }^{22}$ Unfortunately, most studies ignore this factor. The present study indicated that preemptive FA resulted in less sufentanil consumption during surgery than postoperation FA, which is maybe the result of the prevention of the establishment of central sensitization. The reason why there was no difference in VAS of postoperation between Groups B and C may ascribe the difference in sufentanil consumption during surgery.

There are two phases - incisional and inflammatory (reaction to the damaged tissue) - in surgery-induced central sensitization. It is suggested that 
as inflammatory injury plays dominant role, antinociceptive protection provided by preemptive treatment should extend into the postoperative period to cover the inflammatory phase; otherwise, it is ineffective as in the rat paw incisional model. ${ }^{1}$ The analgesic effect of FA would begin 30 min after administration, with an elimination half-life of 6 h. ${ }^{12}$ We administered FA 15 min before a cervical plexus block in order to make sure that the analgesic effect of FA before the incision and lasted throughout the operation. The analgesic properties of FA can be attributed to their inhibition of COX and the subsequent decrease in prostaglandins in the periphery. ${ }^{23}$

A nerve block is one of the modalities of preemptive analgesia studied. ${ }^{24}$ All patients in the present study, irrespective of the group assignment, received a cervical plexus block before surgery. Probably due to this treatment, the mean pain score was never above 6 (Fig.1). However, the present study did not compare the efficacy of a cervical plexus block as a preventive analgesia and studied only the possible benefits of FA.

The NSAIDs are associated with many adverse effects, including reducing platelet aggregation, renal and gastrointestinal mucosal injury. However, in this study, there was no difference of intraoperative or postoperative blood losses between three groups. Also, no adverse effects on renal and gastrointestinal mucosal injury were found in any of the patients. That may be because of the only single dose infusion. These results are similar to other studies ${ }^{9-11}$.

There are several limitations of the present study. Cervical plexus block may influence the results. Psychosocial characteristics, educational background and preoperative pathology of the patients were not controlled in this study.

In conclusion, preoperative administration of intravenous flurbiprofen axetil reduced analgesic consumption during thyroid gland surgery, but not postoperative pain scores.

\section{Acknowledgments}

This work was supported by the grants from the outstanding youth science foundation (No. JC2007716), the Science and Technique foundation (No. GC06C410) and the important research project of education bureau of Heilongjiang Province of China (NO. 1152hz33).

\section{Conflict of Interest}

There is no potential conflict of financial interests in this study.

\section{References}

1. Kissin I. Preemptive analgesia. Anesthesiology. 2000; 93: 1138-43.

2. Neuss H, Koplin G, Haase O, Reetz C, Mall JW. Preemptive analgesia reduces pain after radical axillary lymph node dissection. J Surg Res. 2010; 162: 88-94.

3. Karaman Y, Kebapci E, Gurkan A. The preemptive analgesic effect of lornoxicam in patients undergoing major abdominal surgery: a randomised controlled study. Int J Surg. 2008; 6: 193-6.

4. Bhatnagar S, Gupta D, Mishra S, Srikanti M, Singh M, Arora R. Preemptive antiemesis in patients undergoing modified radical mastectomy: oral granisetron versus oral ondansetron in a double-blind, randomized, controlled study. J Clin Anesth. 2007; 19: 512-6.

5. Hariharan S, Moseley H, Kumar A, Raju S. The effect of preemptive analgesia in postoperative pain relief--a prospective double-blind randomized study. Pain Med. 2009; 10: 49-53.

6. Reza FM, Zahra F, Esmaeel F, Hossein A. Preemptive analgesic effect of ketamine in patients undergoing elective cesarean section. Clin J Pain. 2010; 26: 223-6.

7. Hoshino K, Sasaki K. Administration of NSAIDs in cases of post operation pain. Pharma Medica. 1993; 11: 141-5.

8. Mikawa K, Nishina K, Maekawa N, Shiga M, Obara H. Dose-response of flurbiprofen on postoperative pain and emesis after paediatric strabismus surgery. Can J Anaesth. 1997; 44: 95-8.

9. Takada M, Fukusaki M, Terao Y, et al. Postoperative analgesic effect of preoperative intravenous flurbiprofen in arthroscopic rotator cuff repair. J Anesth 2009; 23: 500-503.

10. Nishiike $S$, Kato T, Nagai M, et al. Preoperative flurbiprofen for pain prevention after tonsillectomy in adults. Journal of Clinical Anesthesia. 2007; 19: 596-600.

11. Yamashita K, Fukusaki M, Ando Y, et al. Preoperative administration of intravenous flurbiprofen axetil reduces postoperative pain for spinal fusion surgery. J Anesth. 2006; 20: 92-5.

12. Nakayama M, Ichinose $H$, Yamamoto $S$, et al. Perioperative intravenous flurbiprofen reduces postoperative pain after abdominal hysterectomy. Can J Anaesth. 2001; 48: $234-7$.

13. Seltzer Z, Beilin B, Ginzburg R, et al. The role of injury discharge in the induction of neuropathic pain behavior in rats. Pain. 1991; 46: 327-36.

14. Coderre TJ, Katz J, Vaccario AL, et al. Contribution of central neuroplasticity to pathological pain: review of clinical and experimental evidence. Pain. 1993; 52: 259-85.

15. Klein JR, Heaton JP, Thompson JP, et al. Infiltration of the abdominal wall with local anaesthetic after total abdominal hysterectomy has no opioidsparing effect. Br J Anaesth. 2000; 84: 248-9.

16. Vanlersberghe C, Lauwers MH, Camu F. Preoperative ketorolac administration has no preemptive analgesic effect for minor oral surgery. Acta Anaesthesiol Scand. 1996; 40: 948-52.

17. Dahl V, Ernoe PE, Steen T, et al. Does ketamine have preemptive effects in women undergoing abdominal hysterectomy procedures? Anesth Analg. 2000; 90: 1419-22.

18. Møiniche S, Kehlet H, Dahl JB. A qualitative and quantitative systematic review of preemptive analgesia for postoperative pain relief-the role of timing of analgesia. Anesthesiology. 2002; 96: 725-41.

19. Ong CK, Lirk P, Seymour RA, et al. The efficacy of preemptive analgesia for acute postoperative pain management: A meta-analysis. Anesth Analg. 2005; 100: 757-73.

20. Aida S, Baba $H$, Yamakura $T$, et al. The effectiveness of preemptive analgesia varies according to type of surgery: a randomized, double-blind study. Anesth Analg. 1999; 89: 711-6. 
21. Turner GA, Chalkiadis G. Comparison of preoperative with postoperative lignocaine infiltration on postoperative analgesic requirements. Br J Anaesth. 1994; 72: 541-3.

22. Hariharan S, Moseley H, Kumar A, et al. The Effect of Preemptive Analgesia in Postoperative Pain Relief-A Prospective Double-Blind Randomized Study. Pain Med. 2009; 10: 49-53.

23. Ochroch EA, Mardini IA, Gottschalk A. What is the role of NSAIDs in pre-emptive analgesia? Drugs. 2003; 63: 2709-23.

24. Altintas F, Bozkurt P, lpek N, et al. The efficacy of preversus postsurgical axillary block on postoperative pain in paediatric patients. Paediatr Anaesth. 2000; 10: 23-8. 